\title{
Performance Evaluations of Geospatial Web Services Composition and Invocation"
}

\author{
Jianting Zhang, Deana D. Pennington, William K. Michener \\ LTER Network Office, the University of New Mexico, Albuquerque, NM, 8713, USA \\ Contact author email: jzhang@lternet.edu, Phone: 1-505-277-0666
}

\begin{abstract}
:
Geospatial data and analytical functions are essential to geospatial modeling. There are increasing interests in publishing both geospatial data and analytical functions as Web services and use them as the building blocks for domain specific geospatial modeling. While the advantages of using the Web Services technologies have been well recognized and a number of prototype systems have been built to demonstrate the feasibility, very few performance evaluations have been reported in the previous studies. Compared with business data, geospatial data is rich in data types, large in data volumes and complex in semantics. On the other hand, the Web Services technologies are known to have significant overheads with respects to deployment and invocation. The answers to how effective the Web Services technologies can be, and, to what extent they are effective under the typical computation environments for geospatial modeling remain largely unknown.

In this study we have set up an experimental system by deploying several geospatial Web services on top of popular commercial and open source spatial databases and Geographical Information Systems (GIS). The Kepler scientific workflow system is used for geospatial Web services composition and invocation. We have conducted experiments to chain the geospatial Web services into a geospatial model under two data volume levels and two network settings. Our experiments show that the geospatial modeling using the Web Services technologies remains effective in the wired LAN computation environment for data volume as large as 10000 points. However, the same data volume level incurs significant response lags under the wireless WAN computation environment. The experimental results may be used as a guideline for geospatial modeling using the Web Services technologies when performances need to be taken into considerations.
\end{abstract}

\section{Introduction}

Geospatial data processing in distributed environments, an active research topic in spatial databases and GIS, is both theoretically interesting and practically useful. Historically, studies on distributed geospatial data processing have been strongly influenced by the mainstream information technologies, such as the Common Object Request Broker Architecture (CORBA) protocol [1][2] and the HTTP/Web protocols [3][4]. More specifically, several popular standards from the Open Geospatial Consortium (OGC), such as the Web Map Service (WMS), the Web Feature Service (WFS), Web Coverage Services (WCS) and the Web Catalog Services (WCAS), are based on HTTP GET/POST [5]. There are increasing adoptions of the OGC standards to integrate diverse geospatial data sources with applications ranging from archiving library resources [6], military applications [7] to ecological data integration [8] and hydrological modeling [9]. For clarity purposes, we refer the geospatial services based on the OGC standards as OGC-Web services.

With the new emerging SOAP based Web Services technologies, we are anticipating that the geospatial data processing community will adopt the W3C Web Services technologies in the near future. As a matter of fact, using WSDL/SOAP/UDDI in conjunction with the OGC Web services have been published as an OGC discussion paper [10] and the needs for OGC Web services to comply with the W3C Web Services technologies have been identified by several studies, e.g. [11]. There are also studies that use the W3C Web Services technologies for geospatial modeling [12][13][14].

The driving motivations for looking for new technologies for geospatial data processing in distributed and heterogeneous computing environments are three folds. First, the monolithic GIS suffer from high initial investment for computer hardware, GIS software and training

\footnotetext{
* Supported in part by NSF grant ITR \#0225665 SEEK and NSF grant ATM \#0619139 CEO:P—COMET.
} 
users. Second, more and more geospatial functionalities are being provided by diverse GIS packages and spatial database systems, in addition to native implementations using programming languages. Subsequently geospatial analytical components are developed using GIS scripting languages, extended SQL query languages and native APIs. The diverse implementations make the integration of these models a non-trivial task even on a single machine [12][14]. Third, while currently most geospatial data and models are managed locally, there are increasing needs to integrate theses distributed data and models for large-scale geospatial modeling in a dynamic manner.

The W3C Web Services technologies provide a published interface to data or programs by using Web Service description Language (WSDL). Using Web Services for geospatial data handling has attracted increasing research and application interests for various reasons. First of all, WSDL is an XML based language and is platform independent. Second, the elements defined by WSDL enable a program to understand how it can interact with a Web service in a systematic way. Third, and perhaps most importantly, Web Services technology and WSDL are the de-facto industrial standards with wide acceptances. From geospatial data processing perspective, the highly successful Web/Internet GIS [15] is a special case of Web services where raw data is transformed into a browser displayable format. However, Web Services technology is a step further which virtually allows any geospatial processing methods/programs to be exposed to the Web.

While we can implement a single analytical step as a Web service, for geospatial modeling, we need to compose multiple Web services into geospatial models. OGC recently list Geo Processing Workflow (GPW) as one of the threads in its phased 4 Web Services initiative [16]. However, the initiative is in progress and the details are currently unavailable.

Visual modeling approaches (such as drag-drop-connect), similar to the way ModelBuilder of ESRI ArcGIS [17] and Spatial Modeler of ERDAS Imaging [18] work in their host environments, are desirable to nonprogrammer users. They are mostly useful for composing simple workflows and chaining analytical modules for direct execution purposes using the system's built-in modules. To our knowledge, they currently do not support W3C Web services. Several scientific workflow technology shares many similarities with the visual modeling approaches. Many scientific workflow systems, such as Taverna [19], Triana [20] and Kepler [21], support integrating distributed and heterogeneous computing resources using the $\mathrm{W} 3 \mathrm{C}$ Web Service technologies. Our previous studies have demonstrated the feasibility of using the Kepler scientific workflow systems to build geospatial models by geospatial Web service compositions [13][14].

While the W3C Web Services technologies are promising for the integrations of distributed geospatial data processing, most of the existing studies focus on the "feasibility" studies, i.e., building prototypes using Web services and execute the composed workflow to achieve domain specific goals, few of them touch the "effectiveness" aspects, i.e., how effective the Web Services technologies can be, and, to what extent they are effective under the typical computation environments for geospatial modeling. To this end, we have set up an experimental system by deploying several geospatial Web services on top of popular commercial and open source spatial databases and GIS. The Kepler scientific workflow system is used for geospatial Web services composition and invocation. Experiments have been conducted to evaluate the effectiveness of geospatial Web services composition and invocation.

The rest of the paper is arranged as follows. Section 2 introduces the architecture of the prototype system in which the experiments are performed. Section 3 describes the geospatial Web services and the workflow used in the experiments. Section 4 presents the experimental results and some discussions. Finally section 5 is the summary and future work directions.

\section{Prototype System Architecture}

The prototype system consists of the Kepler scientific workflow system for composing and executing geospatial scientific workflows (models) and the Web service endpoints for providing geospatial data and geospatial analytical functions. The architecture of the prototype system is shown in Fig. 1. Key components in the architecture are further introduced below.

\subsection{The Kepler Scientific Workflow System}

Kepler builds upon the mature, datafloworiented Ptolemy II system (Ptolemy, [22]). Ptolemy controls the execution of a workflow via so-called directors that represents models of 
concurrent computation. Individual workflow steps are implemented as reusable actors that can represent data sources, sinks, data transformers, analytical steps, or arbitrary computational steps. An actor can have multiple input and output ports, through which streams of data tokens flow. Additionally, actors may have parameters to define specific behavior. Ptolemy supports hierarchical workflow modeling through the concept of composite entity. Kepler inherits and extends these advanced features from Ptolemy and adds several new features for scientific workflows, such as ontology-based data and actor searching, semantic type checking and advanced object management for distributed execution of workflows. Kepler/Ptolmey provides a graphical user interface for interactively composing scientific workflows and allows step-by-step executions. We found both features are especially appealing to nonprogrammer scientific users and refer readers to [23] for more formal presentations of Kepler.

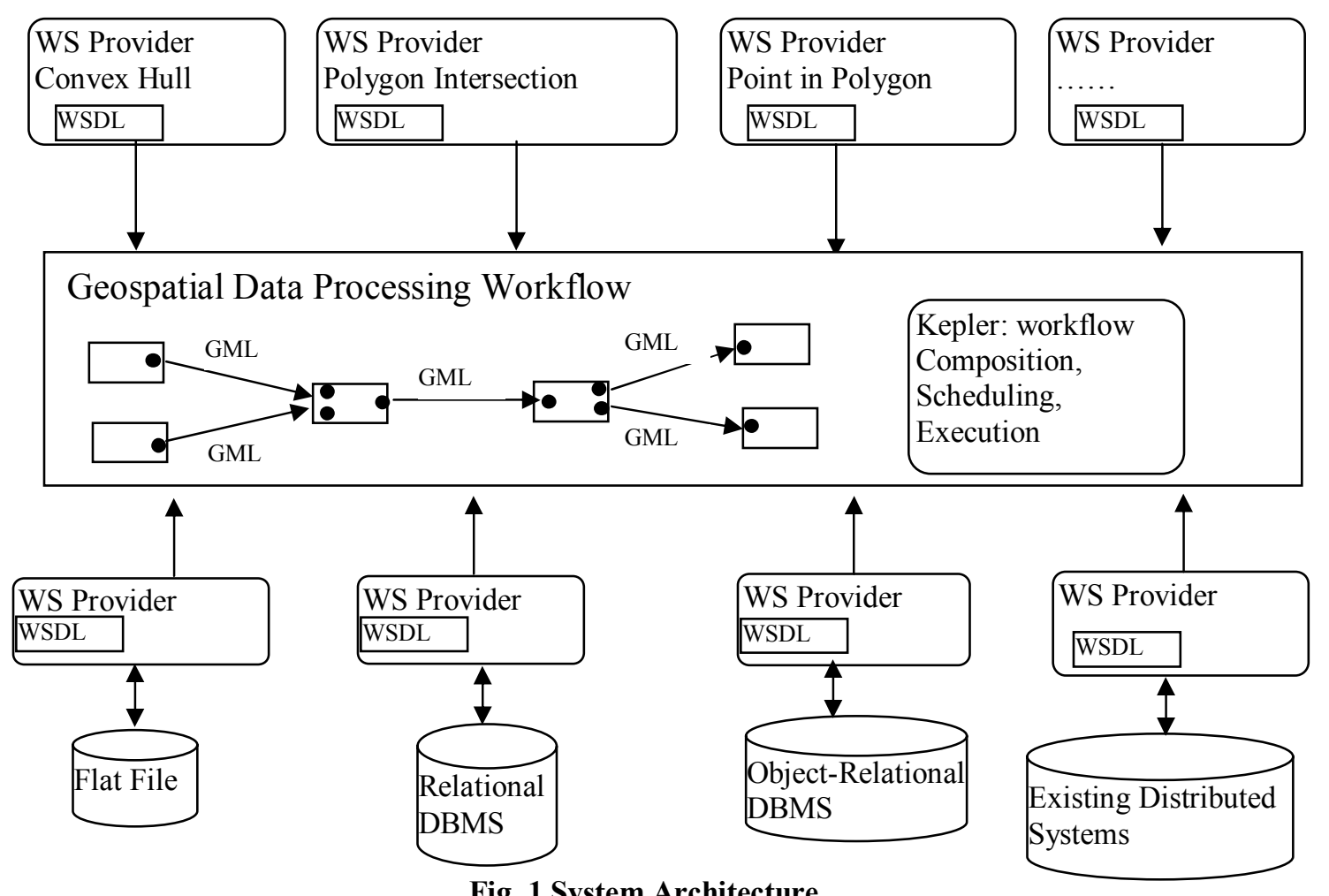

\subsection{The Web Service Actor}

The Web Services actor in Kepler acts as a proxy between the workflow system and the Web Service endpoints. There are two steps to use a Web Service actor in Kepler. The first step is to specify the URL of the WSDL of a Web Service. The Web Service actor parses the WSDL URL and retrieves available methods (operations) and their input/output types (portTypes) declared in the WSDL document. In the second step, users are required to select a method from a dropdown list. After the selection, Kepler maps the input/output types to its type system, instantiates the ports based on the inputs/outputs and adds the ports to the actor. Users then can connect the ports of an actor to the ports of other actors. Note Kepler actor port is different from the port definition in WSDL.

When a Web Service actor is executed (or "fired" in Ptolemy/Kepler terminology), the actor collects all the data tokens from the input ports and transforms them into values of primitive XML data types. The values are further transformed to Java objects and put into an object array. The object array is then used in client API of a Web Services engine (such as Apache Axis) to invoke the Web service endpoint. Finally the invocation result is mapped back to Kepler output ports by setting their output data tokens. Currently the Web Service actor supports primitive XML data types and their 
array data types only. Supporting complex data types is planned.

\subsection{GML Tunneling in WS Invocation}

Geographical Markup Language (GML, [24]) has gained much popularity in geospatial data exchanges in recent years. Major GIS vendors now begin to support GML. We are expecting that when GML become the ISO International Standard (IS) 19136 in the near future [25], GML can serve as bridges between both commercial and open source geospatial data processing systems (Web services in particular). In the prototype system architecture, GML strings are embedded in SOAP messages to be passed through Web services endpoints, which we term as GML tunneling as detailed below.

Currently Axis Web Services engine requires type matching of all parameters of a Web service before it can be invoked. Although it is possible to generate stub codes from the WSDL of a Web service to ensure type matching by source code compiling (i.e., if types do not match codes will not compile), this two-step static binding approach virtually requires a user to be a programmer which might impose too much burden to scientific users. Dynamic or stubless Web service invocation [26] may be more desirable under the application context. However, invoking Web services in a program that has complex data types without constructing Java instances of such data types is not supported in the current Axis architecture. According to Axis, in order to invoke Web service with complex data types, the mappings between the names of the complex data types that can be put in SOAP messages and some Java classes has to be established when the Web service is deployed (serialization/deserialization). This prevents the Web services that have already been deployed from adjusting themselves to the incoming parameter data types in dynamic SOAP messages. To get around the restriction of Axis architecture, we propose a tunneling approach for geospatial Web services invocation, i.e., only GML strings and primitive XML data types will be used in Web service interfaces while leaving the interpretation of GML strings to the geospatial processing Web services.

The term "tunneling" originally comes from computer network domain where a data packet is put inside another. The outer packet is used for transportation between two communication points according to a certain common protocol while the inner packet carries the protocol that can only be understood between the two points and is irrelevant to others. In our implementation, the string data type defined by SOAP is used as the protocol of outer packets which is primitive and can be understood by all Web services. The GML is used as the protocol of the inner packet and can only be understood by the calling party and the functional part of a geospatial processing Web service. As discussed in [14], a caution must be kept in mind that the strategy essentially shifts the responsibility of ensuring the compatibilities among the inputs and outputs of Web services to the Web service endpoints.

\section{Geospatial Workflow and Geospatial Web Services}

We use the following scenario in species distribution analysis as an example for performance evaluations purposes. The analysis involves finding all the occurrences of species $\mathrm{C}$ that are within the intersection of the convex hulls of the occurrences of species A and species B. The three species occurrences data can be stored in flat files, relational databases or object-relational databases. There are a few geospatial analytical functions required in the modeling: finding convex hull of a set of points, polygon intersection and point in polygon test. The example is a reimplementation of our previous study reported in [12].

In the model, we assume the occurrence databases (point data) are located in three systems: species A data is in flat file format, species B data is managed by SQL Server 2005 and species C data is managed by Oracle $10 \mathrm{~g}$ with Spatial option. These three data sets are published as Web services. The latitude and longitude values of the occurrence data are stored as two separate columns in the flat file and they are stored as two fields of real type in a SQL Server 2005 table. To utilize Oracle's spatial functions, we store the latitude and longitude values as an MDSYS.SDO_GEOMETRY type. While transforming flat file and relational table to/from GML strings is left to user implementations, Oracle supports both transforming GML to database and transforming a query result set into GML in two steps. First, GeometryAdapter (8i) or JGeometry $(10 \mathrm{~g})$ is used to map between a geometry object and a SQL structure in Oracle. Second, an Oracle Geometry object is mapped to/from a GML element.

There are seven Web services actors used in the workflow (c.f. Fig. 2). Three Web services to get data points in GML format from the flat file (FlatFileWS), SQL Server database 
(SQLSvrDBWS) and Oracle database (OracleDBWS), respectively. There are two copies of the convex hull Web service to compute the convex hulls of the occurrences of species A and B (ConvexHullWS1 and ConvexHullWS2). The Web service to compute the intersection of the two convex hulls is called PolyIntersectionWS and finally the Web service to perform point in polygon tests is called PInPWS. The three data source Web services are data-intensive. The convex hull Web services as well as the point in polygon test Web service are both data and computation intensive. Theoretically, the polygon intersection Web service is both data and computation intensive as well when the numbers of points in the polygons are large. However, since the number of points in the convex hulls (polygons) derived from randomly distributed point data sets are limited (usually less than 100 based on our experiments), the data communication and computation demands are considerably lighter than other Web services.

All the Web services are deployed at a Dell Latitude D610 laptop with $1 \mathrm{G}$ memory running Apache Tomcat 5.0.28 and Axis 1.2. We choose to deploy the Web services at a laptop for testing due to institutional security policies. However, since the laptop was wired to a local area network and hooked to a continuous power supply, it functions pretty similarly to a regular Web server. The geospatial model/workflow is designed to perform experiments under two types of network settings on two different data volume levels which give totally four experimental scenarios. The first network setting is executing the workflow at a wired gigabyte Local Area Network (LAN) where the Web server is located. The client machine running Kepler is a Dell OptiPlex GX270 desktop with $1 \mathrm{G}$ memory. We term the setting as Wired $+L A N$. The second network setting is executing the workflow at a wireless network environment where the available bandwidth is much smaller and the communication is more error-prone. The client machine running Kepler is a Dell Inspiron 6000 laptop with 768M memory. An $11 \mathrm{M}$ bps Netgear wireless router is used to connect the client machine to the Web server machine through a regional public Internet Service Provider (ISP) providing a maximum data communication rate of $6 \mathrm{M}$ bps. The network setting is similar to a Wide Area Network (WAN) environment and we term the setting as Wireless $+W A N$. We set the number of data points in each species occurrence data sets at two levels (i.e., data volume levels): 1000 points and 10000 points. The first level represents the normal data volume of present practices in the research field based on the authors' experience. The second level represents possible data volume in the foreseeable future based on the authors' estimation. The experiment scenarios play important roles in the implementations of the Web services as detailed below.

We use JUMP GIS [27] as the representative example of open source GIS software and Oracle Spatial [28] as the representative example of commercial spatial databases to demonstrate how their spatial analytical functionalities can be integrated by using Web Services and scientific workflow technologies. JUMP GIS supports computing the convex hull from a set of points using its API. Oracle supports computing the convex hull from a table storing the points using its extended SQL query statements (SDO AGGR CONVEXHULL and SDOAGGRTYPE). We choose to use JUMP GIS to implement the convex hull Web service to avoid converting a GML string to a table for each Web service invocation. Both of them support polygon intersection in a similar fashion. However, the polygon intersection in Oracle Spatial (SDO_INTERSECTION) can directly work on two geometric objects and does not require aggregation of the geometric objects from a table or a view as in generating convex hull. We thus choose to implement the polygon intersection Web service using Oracle Spatial. Similar to the considerations in implementing the convex hull Web service, we again choose to use JUMP GIS to implement the point in polygon test Web service to avoid constantly creating, populating and dropping temporal tables.

\section{Results and Discussions}

The composed workflow is shown in Fig. 2. The workflow is quite straightforward except that all the intermediate GML data strings are linked to a same GML Displayer actor for visualization which makes the connection looks a little bit complex. We purposely set the input port of the GML Displayer actor to accept multiple inputs (or multiport in Ptolemy/Kepler terminology [22]) to visualize all the data sets in one map. We want to mention that while the Synchronous Data Flow (SDF) director [22] is used in the experiment which schedules all the actors to be executed sequentially, we could have used the Process Network (PN) director [22] to parallelize the two convex hull generation processes. This is especially useful when multiple copies of the convex hull Web services are deployed in multiple Axis servers and the data volume level is high. 
The experiments show that the times to invoke the Web Services actors within the Kepler and the times to pass data tokens among the Kepler ports are negligible and they are excluded from further discussions. The Kepler scientific workflow system has been proven to be effective in Web services composition, validation and interactive invocation.

We also measure the invocation times of the seven geospatial Web services individually. The invocation times under the four scenarios described previously, i.e., two network settings by two data volume levels, are listed in Table 1. We also plot the results in Fig. 3 to better interpret the results. Table 1 shows that for data intensive Web services (FlatFileWS, SQLSvrDBWS and
OracleDBWS), the Web services invocation times are considerably affected by the data volumes. In particular, the data volumes at the level 2 is about 10 times of those at the level 1 and the invocation times increase roughly proportionally. Table 1 and Fig. 3 show that the invocation time at the Wirless $+W A N$ network setting can be several times larger than that at the Wired $+L A N$ for a same Web service which indicate the significant overheads of distributed computing. We also have observed that the invocation time of a Web service also can be several times larger than the computation time of a native implementation (not shown in table 1) which indicates the significant overheads of integrating heterogeneous computing resources using the Web Services technologies.

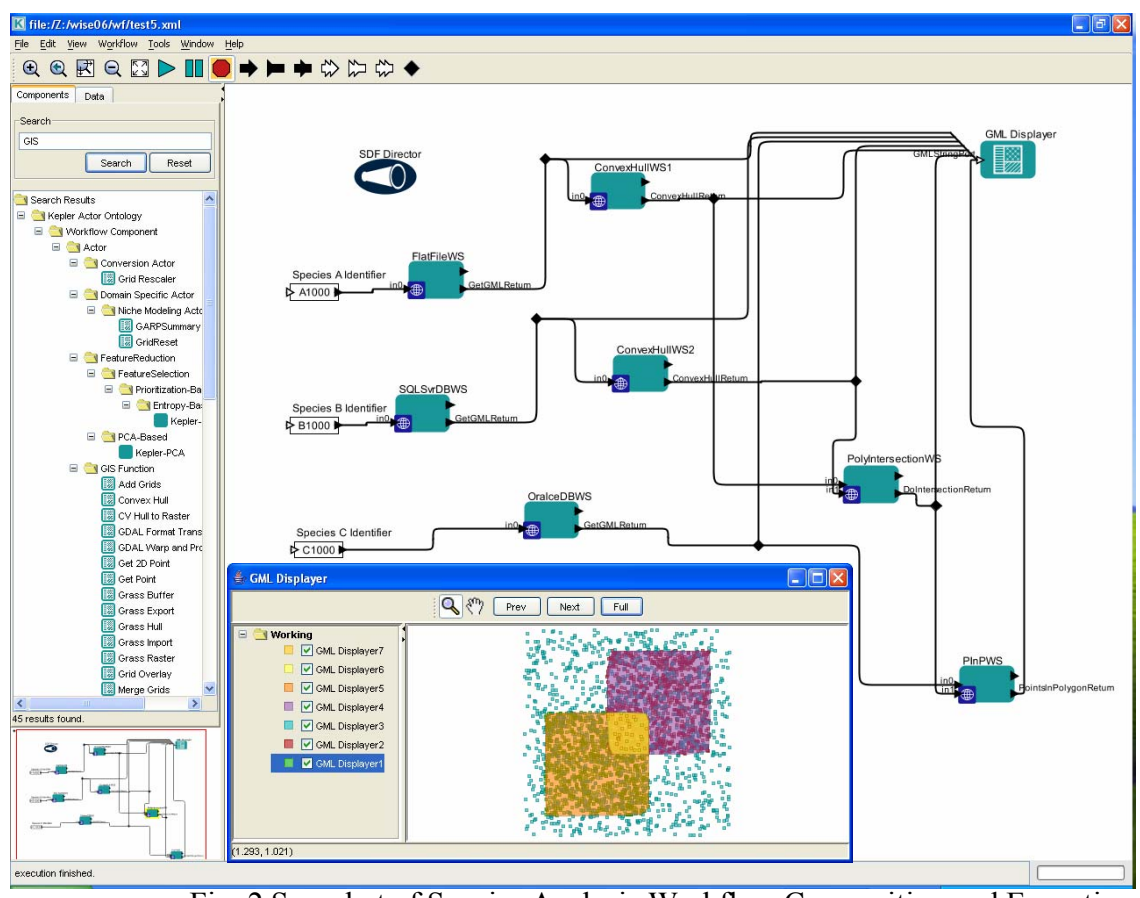

Fig. 2 Snapshot of Species Analysis Workflow Composition and Execution

From Table 1 we can see that largest invocation time among the seven Web services under data volume level 1 and the Wired $+L A N$ setting is about 2.5 seconds and the total invocation time is about 7 seconds. These numbers are increased to 9 seconds and 36 seconds, respectively, under the Wireless $+W A N$ setting for the same data volume level. The largest invocation time and the total invocation time are further increased to 26 seconds and 77.6 seconds, respectively, under the data volume level 2 and the Wired $+L A N$ setting. The response times under the first scenario (data volume level 1 and the Wired $+L A N$ setting) are perfectly acceptable from a user perspective. The response times under the second scenario (data volume level 1 and the Wireless $+W A N$ setting) and the third scenario (data volume level 2 and the Wired $+L A N$ setting) are generally acceptable as well. However, the response times under the last scenario, i.e., data volume level 2 and the Wireless $+W A N$ setting, which are 98.7 seconds for that largest invocation time and 356.4 seconds for the total invocation time, while they may be acceptable for noninteractive applications, are questionable with respect to the effectiveness of the response times of the corresponding Web services and the whole geospatial workflow from a user perspective.

Based on the results, while we advocate for using Web Services technology for geospatial 
modeling in distributed and heterogeneous computing environment due to the excellent capabilities of interoperability brought by the technologies, we suggest taking data volume and

Table 1 Invocation Time of the Web services under the Four Experiment Scenarios

\begin{tabular}{|c|c|c|c|c|}
\hline $\begin{array}{c}\text { Data } \\
\text { Volume } \\
\text { Level }\end{array}$ & $\begin{array}{c}\text { Web } \\
\text { Service }\end{array}$ & $\begin{array}{c}\text { Data Size } \\
\text { (Bytes) }\end{array}$ & $\begin{array}{c}\text { Time } \\
\text { Wired+LAN } \\
\text { (Milliseconds) }\end{array}$ & $\begin{array}{c}\text { Time } \\
\text { Wirless+WAN } \\
\text { (Milliseconds) }\end{array}$ \\
\hline \multirow{7}{*}{$\begin{array}{l}\text { Level } 1 \\
(1000 \\
\text { Points) }\end{array}$} & FlatFileWS & 276359 & 563 & 2937 \\
\hline & SQLSvrDBWS & 259346 & 1703 & 4640 \\
\hline & OracleDBWS & 273553 & 2438 & 3047 \\
\hline & ConvexHullWS1 & 2227 & 1016 & 8984 \\
\hline & ConvexHullWS2 & 1678 & 782 & 8032 \\
\hline & PolyIntersectionWS & 1538 & 78 & 704 \\
\hline & PInPWS & 9723 & 672 & 8069 \\
\hline \multirow{7}{*}{$\begin{array}{l}\text { Level } 2 \\
\text { (10000 } \\
\text { Points) }\end{array}$} & FlatFileWS & 2767148 & 5672 & 27563 \\
\hline & SQLSvrDBWS & 2597353 & 6734 & 26937 \\
\hline & OracleDBWS & 2739555 & 10719 & 30234 \\
\hline & ConvexHullWS1 & 2344 & 25969 & 98687 \\
\hline & ConvexHullWS2 & 2170 & 23469 & 94109 \\
\hline & PolyIntersectionWS & 1791 & 141 & 375 \\
\hline & PInPWS & 118036 & 4907 & 78516 \\
\hline
\end{tabular}
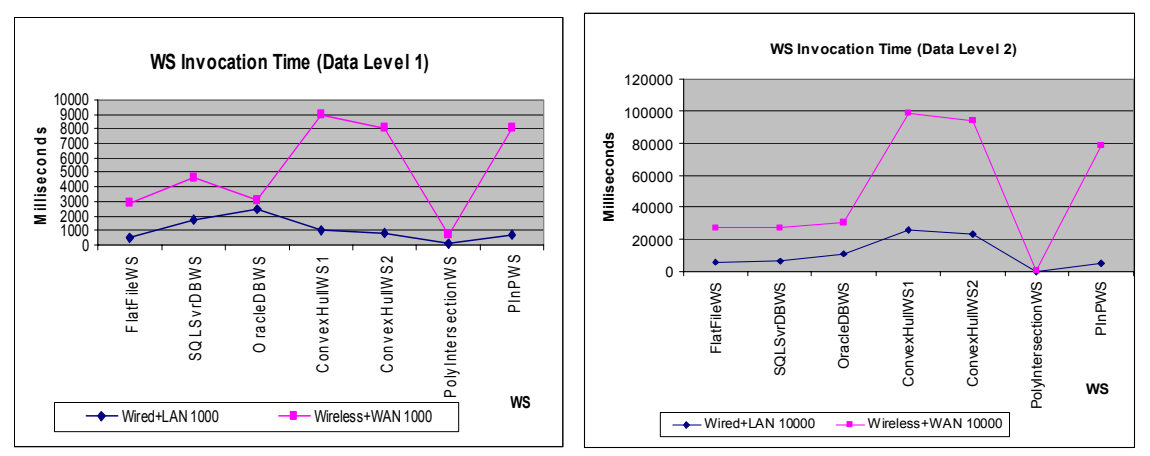

Fig. 3 Comparisons of WS Invocation Time Under the Two Network Settings at the Two Data Volume Levels

\section{Conclusion and Future Work}

In this study, we have built a prototype system to conduct performance evaluations of the composition and invocation of geospatial Web services by using the Kepler scientific workflow system and deploying several geospatial Web services. Our experimental results, in addition to demonstrating the feasibility of integrating distributed and heterogeneous geospatial data sources and analytical functions by using the Web Services technologies, show that the tradeoffs between the conveniences and the overheads of the Web Services technologies are well justified for geospatial data with relative small data volumes. However, the effectiveness of using the network setting factors into considerations when Web services are deployed and invoked. 


\section{Reference}

1. F. Wang, 2000, A Distributed Geographic Information System on the Common Object Request Broker Architecture (CORBA). GeoInformatica, V4, pp. 89-115.

2. M. Preston, P. Clayton and G. Wells, 2003, Dynamic run-time application development using CORBA objects and XML in the field of distributed GIS. International Journal of Geographical Information Science, 17, pp. 321-341.

3. H. Lin and B. Huang, 2001, SQL/SDA: A query language for supporting spatial data analysis and its Web-based implementation. IEEE Transactions On Knowledge and Data Engineering, 13, pp. 671-682.

4. Z. R. Peng, 2005, A proposed framework for feature-level geospatial data sharing: a case study for transportation network data. International Journal of Geographical Information Science, 19, pp. 459-481.

5. OGC Standards (including WMS, WFS, WCS and WCAS), http://www.opengeospatial.org/standards

6. S. P. Morris, 2006, Geospatial Web services and geoarchiving: New opportunities and challenges in geographic information services. Library Trends, 55, pp. 285-303.

7. J. T. Sample, R. Ladner, L. Shulman, E. Ioup, F. Petry, E. Warner, K. Shaw and F. P. McCreedy, 2006, Enhancing the US Navy's GIDB portal with Web services. IEEE Internet Computing, 10, pp. 5360 .

8. M. Frehner and M. Brandli, 2006, Virtual database: Spatial analysis in a Web-based data management system for distributed ecological data. Environmental Modelling \& Software, 21, pp. 1544-1554.

9. C. Kiehle, 2006, Business logic for geoprocessing of distributed geodata. Computers \& Geosciences, 32, pp. 1746-1757.

10. OGC OWS 2 Common Architecture: WSDL SOAP UDDI, http://portal.opengeospatial.org/files/index.php?artif act_id $=8348$, (last accessed 02/24/07)

11. A. Woolf, R. Cramer, M. Gutierrez, K. K. van Dam, S. Kondapalli, S. Latham, B. Lawrence, R. Lowry and K. O'Neill, 2005, Standards-based data interoperability in the climate sciences. Meteorological Applications, 12, pp. 9-22.

12. E. Jaeger, I. Altintas, J. Zhang, B. Ludäscher, D. Pennington and W. Michener, 2005, A Scientific Workflow Approach to Distributed Geospatial Data Processing using Web Services. In Scientific and Statistical Database Management (SSDBM) 2005, 87-90.

13. J. Zhang, D. D. Pennington and W. K. Michener, 2005, Using web services and scientific workflow for species distribution prediction modeling. In Advances in Web-Age Information Management (WAIM) 2005, LNCS 3739, pp. 610-617.

14. J. Zhang, 2006, Tracking Dynamics of Geospatial Phenomena in Distributed and Heterogeneous Environments Using Scientific Workflow and Web
Services Technologies. In Grid and Cooperative Computing (GCC) 2006, 474-481.

15. Peng, Z.-R., Tsou, M.-H., (2003). Internet GIS: Distributed Geographic Information Services for the Internet and Wireless Networks, Wiley.

16. OGC Web Services Initiative, Phase 4 (OWS-4), http://www.opengeospatial.org/initiatives/?iid=199 (last accessed 02/24/07)

17. J. McCoy (2005). Geoprocessing in ArcGIS, ESRI Press.

18. Leica Geosystems (2004). Chapter 16. ERDAS IMAGINE Tour Guides, version 8.7.

19. Taverna, http://taverna.sourceforge.net/ (last accessed 02/24/07)

20. Triana, http://www.trianacode.org/ (last accessed 02/24/07)

21. Kepler, http://www.kepler-project.org/ (last accessed 02/24/07)

22. Ptolemy http://ptolemy.eecs.berkeley.edu/ptolemyII/ (last accessed 02/24/07)

23. B. Ludäscher, I. Altintas, C. Berkley, D. Higgins, E. Jaeger, M. Jones, E. A. Lee, J. Tao and Y. Zhao, 2006, Scientific workflow management and the Kepler system. Concurrency and Computation: Practice and Experience, 18, pp. 1039-1065.

24. OGC Geographical Markup Language (GML) 3.1, $\mathrm{http} / / /$ portal.opengis.org/files/?artifact_id $=4700$ (last accessed 02/24/07)

25. W. Kresse, K. Fadaie, 2004. ISO standards for geographic information, Springer.

26. P. A.Buhler, C. Starr, C., et al., 2004, Preparing for Service-Oriented Computing: A Composite Design Pattern for Stubless Web Service Invocation. In International Conference on Web Engineering (ICWE) 2004, LNCS 3140, 603-604.

27. Unified Mapping Platform (JUMP), http://www.vividsolutions.com/jump/ (last accessed $02 / 24 / 07)$

28. Oracle Spatial, http://www.oracle.com/technology/products/spatial/ (last accessed 02/24/07) 